\title{
Avaliação de Mecanismos de Seleção Dinâmica de Modulação em Redes IEEE 802.15.4g com Diferentes Estratégias de Estimação de Qualidade de Enlace
}

\author{
Diego Miranda de Medeiros, Emerson Brasil Gomes, Ruan Delgado Gomes e Paulo Ribeiro Lins Júnior
}

\begin{abstract}
Resumo-Tanto a atenuação por multipercurso, quanto o sombreamento e as interferências, são penalidades significativas para o desempenho das redes sem fio, principalmente em ambientes restritivos como, por exemplo, os industriais. Para lidar com as restrições do canal de comunicação sem fio, este trabalho propõe o uso de diversidade de modulação na camada de enlace, em que diferentes modulações podem ser usadas para transmitir diferentes réplicas de um pacote ou pacotes subsequentes. Foram propostas duas novas estratégias para estimação de qualidade de enlace, que foram aplicadas a um mecanismo de seleção adaptativa de modulação em redes IEEE 802.15.4g, proposto em [1]. As avaliações foram realizadas com base em um dataset obtido a partir de um experimento realizado em ambiente industrial usando as modulações do IEEE 802.15.4g SUN (SUN-FSK, SUNOQPSK e SUN-ODFM). Os resultados mostraram que as novas estratégias de estimação de qualidade de enlace propostas neste artigo permitiram uma melhoria de confiabilidade na rede, em comparação com a estratégia originalmente proposta em [1].
\end{abstract}

Palavras-Chave-Diversidade de Modulação, IEEE 802.15.4g, Redes de sensores sem fio industriais

\section{INTRODUÇÃO}

Com a chegada da quarta revolução industrial (indústria 4.0), a demanda por monitoramento e controle de processos industriais vem aumentando de forma significativa [2]. O monitoramento é feito, tipicamente, utilizando uma rede de sensores cabeada. Apesar de apresentarem maior confiabilidade, redes cabeadas têm limitações associadas a custo e escalabilidade [3].

A alternativa mais direta a esse tipo de rede é o uso Redes de Sensores Sem Fio (RSSFs), que possuem vantagens em relação às redes cabeadas, uma vez que se destacam no que diz respeito ao baixo custo associado a instalação, sua flexibilidade de expansão, a capacidade de alteração topológica, e à facilidade na implementação e manutenção [4]. Além dessas vantagens, RSSFs possuem a capacidade de auto-organização, possibilidade de processamento local e a instalação em locais de difícil acesso ou até mesmo em objetos que se movem constantemente [5].

Diego Miranda de Medeiros é aluno do IFPB, Campina Grande, e-mail: diego.medeiros@academico.ifpb.edu.br. Emerson Brasil Gomes é doutorando na UFCG, e-mail: emersonjpa@gmail.com. Ruan D. Gomes é professor do IFPB, João Pessoa, e-mail: ruan.gomes@ifpb.edu.br. Paulo Lins Júnior é professor do IFPB, Campina Grande, e-mail: paulo.lins@ifpb.edu.br. Todos são membros do Grupo de Pesquisa em Comunicações e Processamento de Informação - GComPI. Este trabalho foi parcialmente financiado pelo IFPB (Interconecta 2020) e pelo CNPq (421461/2018).
No caso das RSSF Industriais (RSSFIs), os sensores são instalados em equipamentos industriais, que podem realizar tarefas como o monitoramento de temperatura, pressão e vibração [6]. Geralmente, essas informações são encaminhadas através do canal sem fio até uma central, que realiza o monitoramento. Com a coleta dos dados, é possível fazer previsões de falhas, observar máquinas que apresentem algum tipo de problema, evitando assim maiores prejuízos [4].

Apesar das vantagens, as RSSFIs carregam um conjunto de desafios que devem ser levados em conta. O principal deles é a falta de confiabilidade no canal de comunicação, sujeito à interferências eletromagnéticas, e problemas de atenuação relacionados a sombreamento e multipercurso [5].

No ambiente industrial, esses problemas são agravados. Nesse tipo de ambiente, é possível encontrar várias fontes de interferências, principalmente de outros equipamentos que utilizam comunicação sem fio [7]. Além disso, existem vários objetos metálicos e, em grade parte, esses objetos estão se movendo, podendo assim ocasionar ainda mais atenuação do sinal nesse tipo de ambiente [8]. Esses problemas dificultam a garantia de qualidade de serviço (QoS). Aplicações industriais requerem alta taxa de entrega de pacotes e alta disponibilidade, garantir esse requisitos é de suma importância para implementação de uma RSFFI [5].

Esses problemas induzem uma penalidade significativa no desempenho das redes sem fio. Uma possibilidade para mitigar esses efeitos, e prover uma melhor confiabilidade, é fazer uso de mecanismos de diversidade como, por exemplo, diversidade de canal ou de rota [9]. A diversidade é uma técnica usada para compensar os danos causados pela atenuação no canal, sem a necessidade de aumentar a potência ou a largura de banda [10]. Em [1] foram propostas estratégias que usam diferentes esquemas de modulação para transmitir diferentes réplicas de um único pacote ou de pacotes subsequentes. Os resultados mostraram que essa técnica melhora de forma significativa a confiabilidade em redes sem fio que operam em bandas Sub$\mathrm{GHz}$ (868 MHz), apesar de apenas um estimador de qualidade de enlace baseado no transmissor ter sido implementado e avaliado em conjunto com o mecanismo de diversidade.

Portanto, o objetivo deste artigo é avaliar diferentes estratégias de estimação de qualidade de enlace aplicadas ao mecanismo de seleção dinâmica de modulação proposto em [1], para redes IEEE 802.15.4g. Foram implementados e avaliados dois novos mecanismos de estimação de qualidade de enlace, 
baseados em cálculos realizados no receptor, para decidir a melhor modulação a ser usada em determinado momento. Com os novos estimadores de qualidade de enlace foi possível obter um aumento de confiabilidade em relação à versão original do mecanismo.

\section{VISÃo GERAL DO PADRÃO IEEE 802.15.4G}

O padrão 802.15 .4 foi lançado inicialmente em 2003 e é destinado para aplicações de redes de sensores sem fio de baixa potência, baixa taxa de transmissão, baixo consumo e baixa complexidade. Em 2012, foi lançada a emenda 802.15.4g, que definiu três novas modulações direcionadas a aplicações SUN (Smart Utility Network): SUN-FSK, SUN-OPQSK e SUNOFDM.

A modulação SUN-FSK possui vantagens em relação a eficiência energética e baixa complexidade de implementação. A modulação SUN-FSK também é compatível com vários sistemas legados. Três modos de operação diferentes são definidos para cada faixa de frequência, e em cada modo são definidos parâmetros de modulação e canal, como o tipo de modulação (2-FSK que fornece uma taxa de transmissão de dados de 50 a 100 kbps e 4-FSK que fornece uma taxa de até $200 \mathrm{kbps}$ ), o espaçamento do canal e o índice de modulação [11].

O SUN-OQPSK emprega o DSSS (Direct Sequence Spread Spectrum), que permite uma melhor resistência a interferências. Essa modulação pode ser configurada para oferecer uma taxa de transmissão que varia de 6,25 kbps até $500 \mathrm{kbps}$, dependendo do fator de espalhamento [11].

O SUN-OFDM foi incorporado visando obter altas taxas de dados e longo alcance, ao mesmo tempo em que consegue lidar com problemas de interferência causados por múltiplos caminhos. Com o OFDM é possível transmitir a mesma informação por subportadoras diferentes. Apesar dessas vantagens, o OFDM carrega algumas dificuldades, pois sua implementação é mais complexa e é necessário um processamento rigoroso, ocasionando um maior consumo de energia. O SUN-OFDM pode fornecer uma taxa de transmissão de 50 a $800 \mathrm{kbps}$ [11].

\section{DIVERSIDADE DE MODULAÇÃO}

A diversidade de modulação tem como objetivo melhorar a confiabilidade das comunicações usando diferentes modulações para transmitir réplicas de um pacote ou pacotes subsequentes. A ideia principal é que pacotes consecutivos possam ser transmitidos usando duas ou mais modulações, buscando suas vantagens em relação aos efeitos de propagação e interferências. Esse conceito não é novo, mas era limitado em redes sem fio de baixa potência devido à indisponibilidade de transceptores comerciais que suportassem múltiplas modulações. Com o surgimento do padrão IEEE 802.15.4g e a disponibilidade de transceptores, o uso desse mecanismo em redes sem fio de baixa potência pôde ser explorado [12].

Várias estratégias podem ser usadas para implementar esse conceito. Uma parte importante das estratégias é a forma como a qualidade dos enlaces usando cada modulação é estimada. A estimação de qualidade de enlace pode ser realizada de diferentes formas, usando informações disponíveis do lado do transmissor (nó final) ou do receptor (ex: roteador intermediário ou gateway). No nó transmissor, pode ser usado como métrica o ACK Reception Ratio (ARR). O ARR é o resultado da razão entre o número de ACKs recebidos com sucesso e o número de pacotes transmitidos em um determinado intervalo de tempo. Já no lado do receptor, pode ser usado o Packet Reception Ratio (PRR). O PRR é a razão entre pacotes recebidos com sucesso e os pacotes transmitidos de acordo com uma janela especificada.

O uso de diversidade de modulação pode ser de forma fixa, em que é definido o conjunto de modulações a serem usadas e esse conjunto não é modificado no decorrer do tempo nem a frequência de uso de cada modulação. Outra alternativa é a diversidade de modulação adaptativa. Nesse último caso é necessário que a qualidade dos enlaces sejam estimados e, a partir disso, os nós podem decidir qual modulação é melhor em um determinado momento [1].

\section{A. Trabalhos Relacionados}

Em [12] foi realizado um experimento no período de 99 dias, onde 11 nós OpenMote-B foram distribuídos num ambiente industrial real. Foi implementada uma rede de sensores que utiliza uma topologia estrela e os nós foram distribuídos em distâncias que variam de $34 \mathrm{~m}$ a $274 \mathrm{~m}$ em relação ao gateway. Os nós foram configurados para gerar um novo pacote a cada um minuto e enviar usando cada modulação SUN (SUN-FSK, SUN-OQPSK e SUN-OFDM). Um mesmo pacote foi enviado três vezes usando cada modulação, totalizando 9 transmissões. O gateway foi equipado com três transceptores de rádio IEEE 802.15.4g independentes para poder receber pacotes de forma simultânea. Como parâmetro de avaliação, foi utilizado o PDR (Packet Delivery Ratio).

No experimento, foi observado que nenhuma modulação consegue isoladamente fornecer uma alta taxa de entrega de pacote na camada de aplicação para todos os nós e durante todo o tempo. Aplicações industriais necessitam de uma alta taxa de entrega (PDR > 99\%). No experimento, essa taxa foi obtida devido à replicação de pacotes, ou seja, foram feitas 3 transmissões usando cada modulação, totalizando 9 transmissões. Vale salientar que essa técnica pode sobrecarregar a rede, pois o alto número de transmissões aumenta de forma significativa o consumo de energia no nó, limitando sua durabilidade, caso o mesmo seja alimentado por bateria e podendo limitar a escalabilidade da rede. Dando continuidade ao trabalho anterior, em [1] foi proposta a utilização de três estratégias de seleção de modulação adaptativa, denominadas $1 \mathrm{M}, 2 \mathrm{M}$ e $3 \mathrm{M}$, com o objetivo de melhorar a confiabilidade do enlace nas redes IEEE 802.15.4g. A métrica usada para estimar a qualidade do enlace foi o ARR.

A estratégia $1 \mathrm{M}$ usa apenas uma modulação do IEEE 802.15.4g para permitir que os nós transmitam pacotes. Quando a qualidade do enlace cai abaixo de um limiar determinado, a modulação é trocada seguindo uma ordem. A $2 \mathrm{M}$ usa duas das três modulações de maneira intercalada. Se a qualidade de uma das modulações cai abaixo de um determinado limiar, ela é trocada pela terceira modulação que não estava sendo usada. Por último, na estratégia $3 \mathrm{M}$, que 
obteve os melhores resultados, as três modulações são sempre consideradas. Cada nó seleciona uma modulação com base em uma probabilidade, determinada pela Eq. 1.

$$
p_{i}=\frac{\left(1+a_{i}\right)^{w}}{\sum_{k=0}^{k<3}\left(1+a_{k}\right)^{w}},
$$

em que $p_{i}$ é a probabilidade de usar a modulação $i$ (onde 0 é SUN-FSK, 1 é SUN-OQPSK e 2 é SUN-OFDM), $a_{i}$ e $a_{k}$ são valores do ARR para as modulações $i$ e $k$. O expoente $w$ é um fator de peso usado para controlar as diferenças entre os valores de probabilidades calculados.

Se uma tentativa de transmissão falhar, a retransmissão de pacotes não pode usar a mesma modulação. Em vez disso, a modulação a ser usada é selecionada entre as demais de acordo com suas probabilidades. Dessa forma é possível aumentar a diversidade.

Essas probabilidades são calculadas periodicamente com base na qualidade dos enlaces e sempre que um novo valor é calculado para uma das modulações, as probabilidades das outras também são atualizadas. O ARR para uma determinada modulação é calculado com base apenas nos pacotes transmitidos usando esta modulação.

Em [1], além das estratégias de seleção de modulação mencionadas, foram implementadas três estratégias para fins de comparação: Round-Robin, onde os nós usam as modulações de maneira alternada, Random, onde os nós usam as três modulações de forma aleatória com probabilidades iguais e a Best, onde é simulado o maior PDR alcançado caso os nós sempre consigam usar a melhor modulação o tempo todo, essa estratégia representa um limite superior, dada as condições do canal.

\section{Proposta de Estratégia de Diversidade DE ModulaÇÃo AdAptativa}

Foi possível observar que o método de estimação de qualidade do enlace do 3M [1] possui uma limitação. Apesar do ARR conseguir fornecer estimativas de forma rápida sobre a condição do enlace, e não utilizar o canal de comunicação para fazer essa estimativa, o canal sem fio pode apresentar assimetria, de modo que um determinado pacote pode ter chegado no receptor, mas a entrega do pacote ACK pode vir a falhar. Em termos gerais, esse pacote foi entregue, mas como a análise é feita no transmissor, esse pacote é considerado como perdido. Visando investigar novas formas de realizar a estimação de qualidade de enlace no mecanismo $3 \mathrm{M}$, este artigo propõe e avalia duas estratégias alternativas de estimação. A primeira estratégia proposta é chamada de $3 \mathrm{Mnew}$, uma atualização da 3M [1], que realiza no receptor uma análise para calcular a qualidade do enlace. O receptor realiza esta análise usando o PRR, que calcula a razão entre a quantidade de pacotes recebidos pela quantidade de pacotes enviados numa determinada janela para cada modulação. Foram avaliadas janelas contendo entre 6 e 11 pacotes. Após as simulações, observou-se que com uma janela de 9 pacotes, é possível obter um resultado ligeiramente melhor. Na Tabela IV são mostrados os valores de PDR médio global utilizando cada tamanho de janela.
TABELA I

VARIAÇÃO DA JANELA DE PACOTES PARA O CÁLCULO DO PRR.

\begin{tabular}{|c|c|c|c|c|c|c|}
\hline Tamanho janela (pacotes) & 6 & 7 & 8 & 9 & 10 & 11 \\
\hline PDR médio global (\%) & 97,88 & 97,88 & 97,88 & 97,89 & 97,87 & 97,87 \\
\hline PDR máximo (\%) & 99,74 & 99,74 & 99,74 & 99,76 & 99,74 & 99,74 \\
\hline PDR mínimo (\%) & 93,05 & 93,04 & 93,02 & 93,06 & 93,01 & 93,03 \\
\hline
\end{tabular}

Após realizar o cálculo do PRR, é feita uma análise se o valor encontrado é diferente do PRR encontrado na janela anterior, se sim, é enviado um sinal (por meio de um bit no pacote ACK) para o transmissor, que realiza a atualização das probabilidades de uso de cada modulação (de acordo com a Eq.1), considerando o valor do PRR no lugar do valor do ARR para determinar as probabilidades das modulações a serem escolhidas.

A partir da estratégia 3Mnew, foi possível fazer uma avaliação da precisão dos estimadores, com base no dataset descrito em [12]. Para avaliar os estimadores foi calculado o erro médio quadrático, onde o valor mais próximo do zero, indica uma melhor precisão do estimador. Os resultados obtidos mostram que o PRR consegue uma estimação melhor que o ARR. A Tabela II mostra a média dos valores do erro médio quadrático para os 11 nós e para cada modulação.

TABELA II

ERRO MÉdIO QUADRÁTICO PARA CADA ESTIMADOR E MODULAÇÃO.

\begin{tabular}{|c|c|c|c|c|c|}
\hline \multicolumn{2}{|c|}{ FSK } & \multicolumn{2}{c|}{ OQPSK } & \multicolumn{2}{c|}{ OFDM } \\
\hline ARR & PRR & ARR & PRR & ARR & PRR \\
\hline 0,3656 & 0,2831 & 0,4578 & 0,3855 & 0,5083 & 0,4220 \\
\hline
\end{tabular}

A partir das análises realizadas nos estimadores, foi possível observar que, apesar de conseguirem se aproximar dos valores reais, ambos não conseguem realizar a estimação de forma precisa em $100 \%$ do tempo. Chegou-se à conclusão que um estimador mais complexo que conseguisse usar os dois valores para obter a estimação do enlace poderia se sair melhor, se aproveitando das vantagens que cada estimador pode oferecer.

Dessa forma, a segunda estratégia proposta neste artigo foi chamada de $3 \mathrm{Mh}$. Essa nova abordagem é calculada no transmissor, utilizando os valores combinados do ARR e PRR com pesos iguais. Nessa estratégia, é mantida a ideia do $3 \mathrm{M} n e w$, onde o receptor também faz a análise da qualidade do enlace de forma independente, e caso tenha alteração no PRR em relação à janela anterior, o mesmo recalcula as probabilidades, de acordo com a Eq. 2.

$$
\begin{gathered}
p_{i}=\frac{\left(1+\left(\left(\operatorname{arr}_{i} \times 0,5\right)+\left(\operatorname{prr}_{i} \times 0,5\right)\right)\right)^{w}}{\sum_{k=0}^{k<3}\left(1+\left(\left(\operatorname{arr}_{k} \times 0,5\right)+\left(\operatorname{prr}_{k} \times 0,5\right)\right)^{w}\right.} \\
\text { V. Metodologia de Avaliaçẽo }
\end{gathered}
$$

\section{A. Métricas}

As métricas usadas para avaliar o desempenho das estratégias de modulação propostas são o PDR na camada de aplicação e o RNP (Required Number of Packet transmissions). O PDR é calculado pela razão entre pacotes recebidos e transmitidos na camada de aplicação (independente se houve retransmissão ou não), enquanto que a RNP representa o 
número médio de repetições de pacotes antes de uma recepção bem sucedida. A RNP e o PDR são complementares para avaliar a qualidade dos enlaces. Menores valores de RNP indicam que a maioria dos pacotes foi entregue na primeira tentativa de transmissão.

\section{B. Configurações de simulação}

Para avaliar as estratégias mencionadas, foi usado um simulador escrito em Python, descrito em [1], que utiliza o conjunto de dados descritos em [12] para calcular o PDR na camada física de todas as modulações e nós a cada 5 minutos. Com base nos valores de PDR calculados, o simulador determina se cada pacote transmitido e seu reconhecimento (ACK) é entregue ou não. Quando um ACK não for recebido, devido a uma falha em uma das duas transmissões (pacotes ou ACKs), o nó pode retransmitir o mesmo pacote, de acordo com um número máximo de tentativas permitidas. $\mathrm{O}$ simulador foi estendido para dar suporte também às duas novas estratégias de estimação de qualidade de enlace propostas neste artigo. Para obter os resultados descritos neste trabalho, foram feitas 10 simulações para cada nó e obtido o valor médio do PDR e da RNP. Foi permitido o uso de até 6 retransmissões de pacotes, pois acima desse valor o aumento do PDR não é tão vantajoso em relação ao aumento da RNP.

\section{RESUltados}

Nesta sessão são discutidos os resultados obtidos a partir das estratégias de diversidade de modulação adaptativa. Além das duas estratégias propostas neste trabalho, foram usadas as estratégias propostas em [1] como comparativo (1M, 2M, 3M, Random, Round-robin e Best). Para uma melhor análise dos resultados, os nós foram divididos em três grupos, de acordo com a distância $(d)$ entre os nós e o gateway: Grupo $1(d<$ 80 m) - Nós: 5653, 55ad, 55e4; Grupo 2 (80 m $\leq d<150 \mathrm{~m})$ - Nós: 5599, 55dd, 5565, 560b; Grupo 3 ( $d \geq 150$ m) - Nós: 5632, 55b3, 5563, 630a.

As Figuras 1, 2 e 3 mostram o PDR para os grupos 1, 2 e 3 , respectivamente. Através dos gráficos é possível visualizar que a segunda estratégia proposta, $3 \mathrm{Mh}$, consegue um PDR superior em 9 dos 11 nós. Em alguns nós, como o 55ad, OFDM consegue um PDR superior, já no 5565 o OQPSK se saiu melhor. No entanto, levando em conta o PDR global, a estratégia proposta, $3 \mathrm{Mh}$, consegue um PDR superior. Também é possível notar que as estratégias que utilizam os conceitos de diversidade de modulação adaptativa conseguem um PDR superior na maioria dos nós, reforçando que o uso desta estratégia pode melhorar a confiabilidade na comunicação.

A Figura 4 mostra o PDR global das estratégias mencionadas neste trabalho. Como pode-se observar, a estratégia proposta, 3Mh, consegue um PDR global superior.

Como mencionando anteriormente, foi permitido o uso de até 6 retransmissões de cada pacote, caso o mesmo seja perdido. Essa técnica de replicação de pacotes consegue aumentar o PDR, mas pode ter um impacto significativo. Se é necessário realizar várias retransmissões para que um pacote seja entregue, isso significa que o canal de comunicação não está sendo usado de forma eficiente, aumentando o consumo

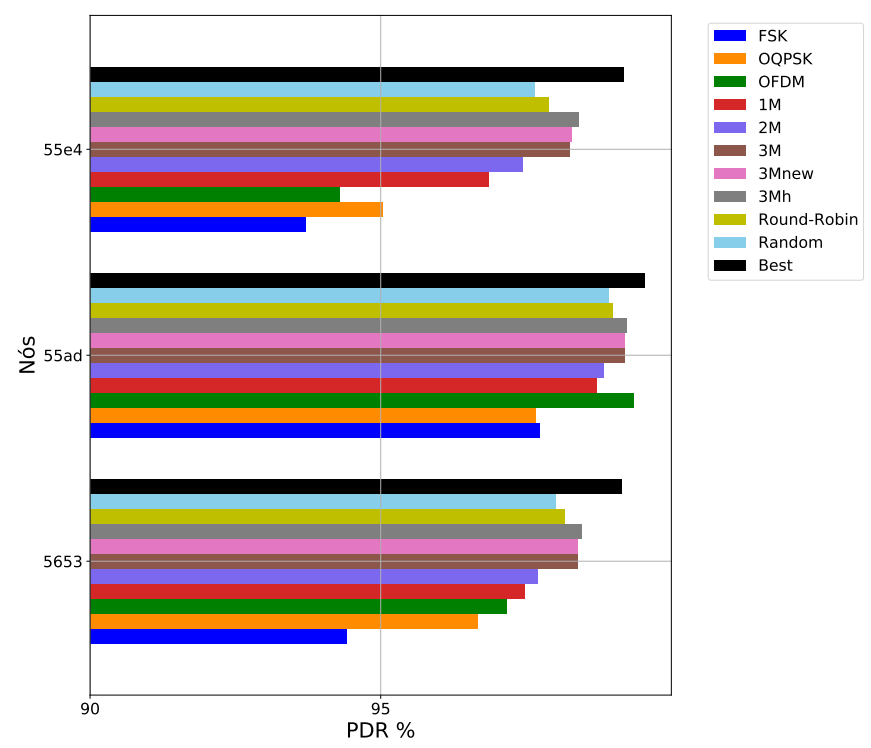

Fig. 1. PDR dos nós que estão no Grupo 1.

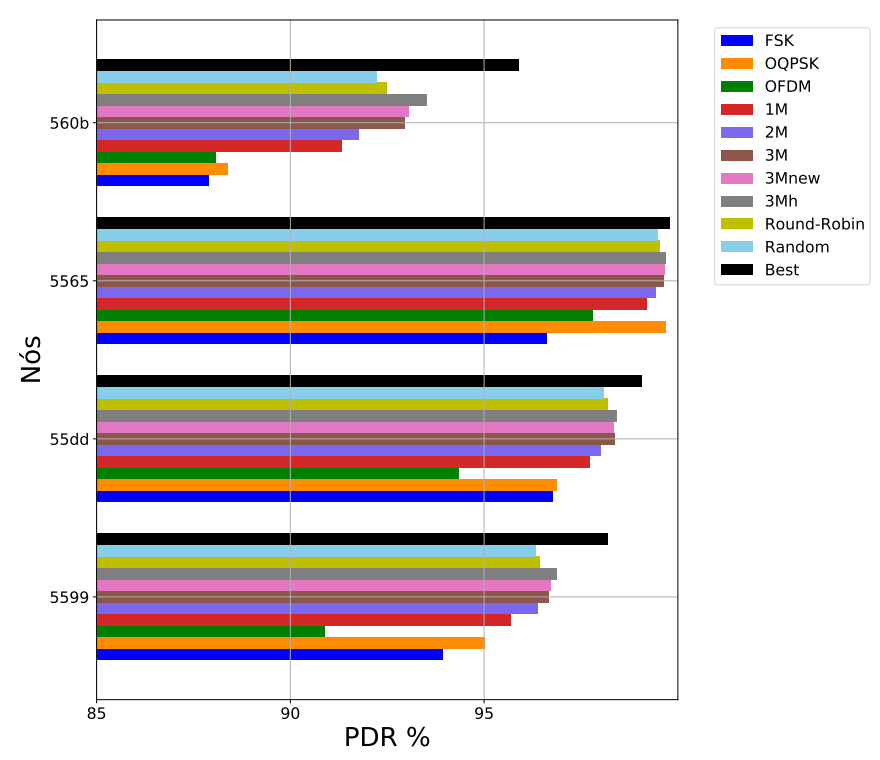

Fig. 2. PDR dos nós que estão no Grupo 2.

de energia nos nós e sobrecarregando a rede. Essa sobrecarga poderá atrapalhar tanto a durabilidade das baterias quanto a escalabilidade da rede, dificultando futuras expansões na rede caso essa sobrecarga seja mantida.

A Figura 5 mostra um comparativo da RNP e o PDR para as diferentes estratégias de diversidade de modulação adaptativa. Cada cor representa uma estratégia de diversidade de modulação e cada ponto representa o número de retransmissões permitidas para essa estratégia, nesse caso, de 1 a 9 . É possível notar que permitir mais retransmissões aumenta de forma significativa o PDR alcançado. Apesar da Round-Robin conseguir um PDR global próximo das estratégias propostas, o valor da RNP dessa estratégia é muito alto, o que implica em maior sobrecarga na rede e maior consumo de energia. 


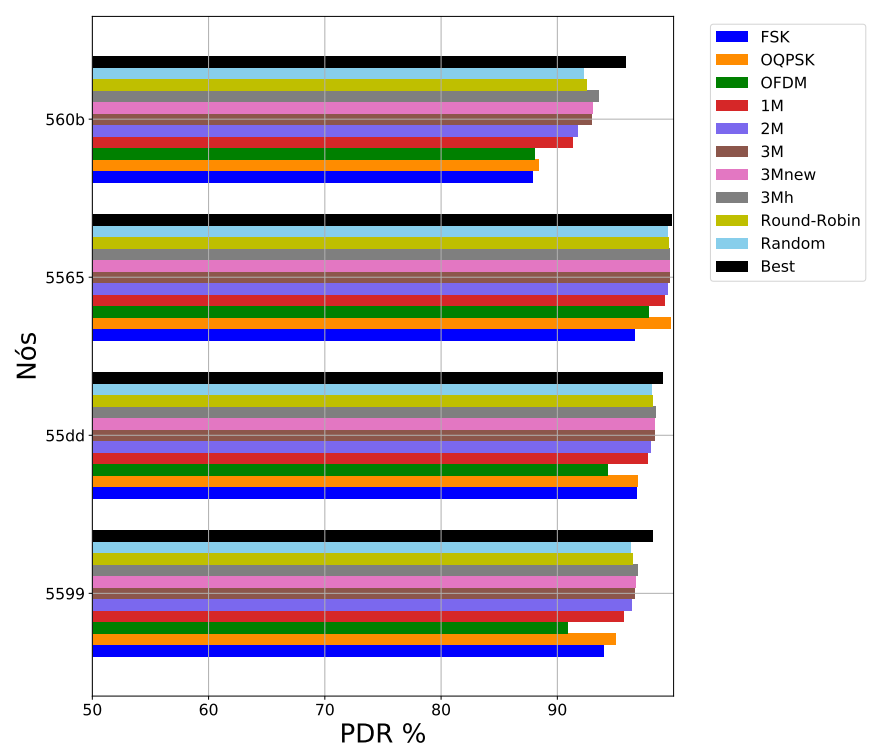

Fig. 3. PDR dos nós que estão no Grupo 3.

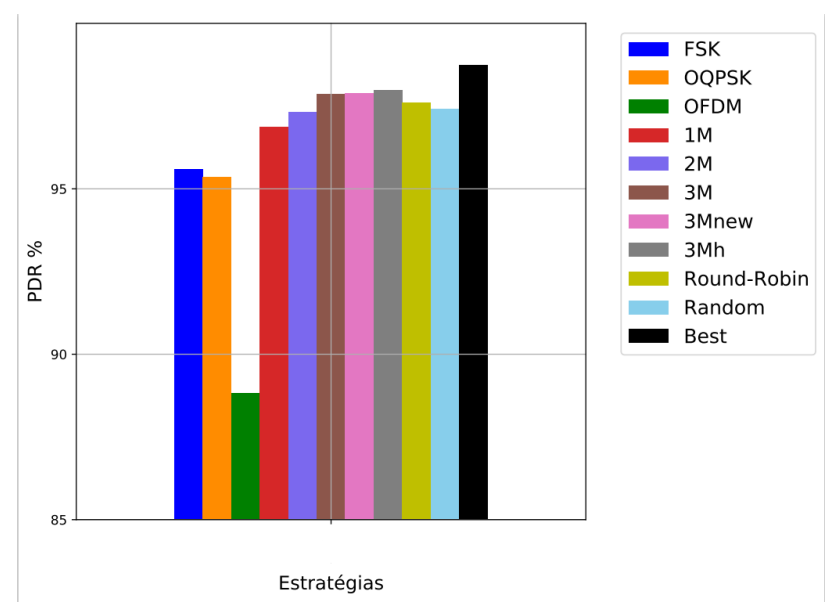

Fig. 4. PDR global

\section{Conclusões e Trabalhos Futuros}

Este artigo propôs duas novas estratégias de estimação de qualidade de enlace aplicadas em um mecanismo de seleção adaptativa de modulação em redes IEEE 802.15.4g. Os resultados obtidos mostram que primeira a estratégia proposta, 3Mnew, consegue obter um PDR ligeiramente superior em 10 dos 11 nós em relação ao estimador do estado da arte (3M). A maior contribuição dessa estratégia foi a possibilidade de obter uma melhor estimação da qualidade do enlace, usando informações calculadas no receptor.

A partir desta estimação mais precisa, foi possível a criação de uma estratégia híbrida, a $3 \mathrm{Mh}$, que utiliza os valores do ARR combinados com o PRR utilizando pesos iguais. A estratégia 3Mh conseguiu um PDR superior em 9 dos 11 nós em relação a 3Mnew, e também um PDR global superior em comparação com as estratégias 3M e 3Mnew. Os códigos fonte, bem como outras análises estão disponíveis em [13].

Como trabalhos futuros, outros mecanismos de estimação

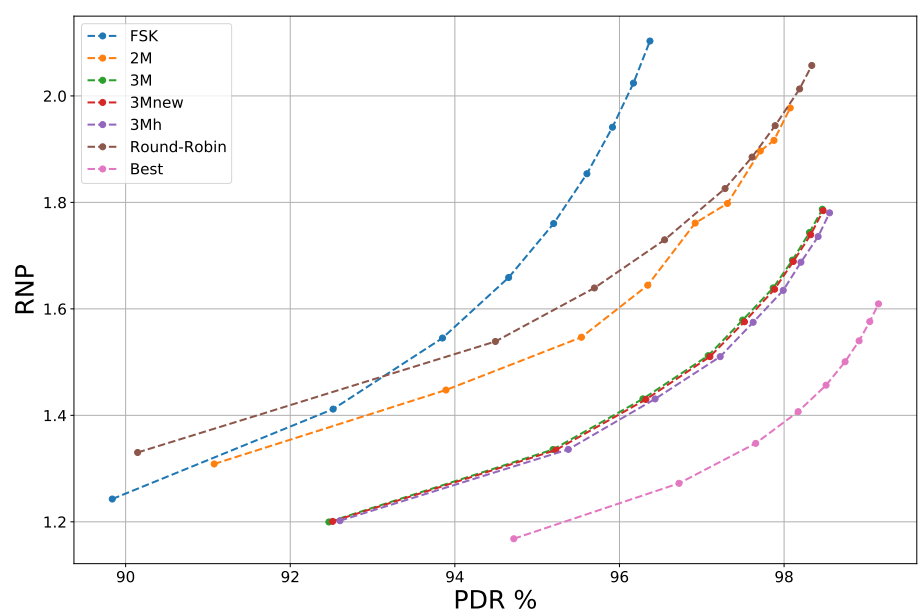

Fig. 5. RNP vs PDR - Cada ponto representa o número de retransmissões de pacotes permitidas para essa estrategia específica, de 1 a 9

de qualidade do enlace poderão ser avaliados, com intuito de obter melhores valores de estimação. Também poderá ser estudado o uso de outros protocolos para a escolha dinâmica de modulação, bem como a realização de novos experimentos em ambientes industriais reais.

\section{REFERÊNCIAS}

[1] R. D. Gomes, P. Tuset-Peiró and X. Vilajosana. "Improving Link Reliability of IEEE 802.15 . 4g SUN with Adaptive Modulation Diversity". In 2020 IEEE 31st Annual International Symposium on Personal, Indoor and Mobile Radio Communications, pp. 1-7. IEEE, 2020.

[2] K. Schwab. A Quarta Revolução Industrial. Edipro, first edition, 2018.

[3] B. Lu and V. C. Gungor. "Online and remote motor energy monitoring and fault diagnostics using wireless sensor networks". IEEE

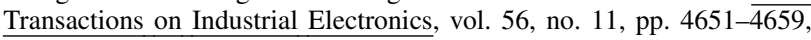
2009.

[4] V. C. Gungor and G. P. Hancke. "Industrial wireless sensor networks: Challenges, design principles, and technical approaches". IEEE Transactions on industrial electronics, vol. 56 , no. 10 , pp. $4258-\overline{4265}$, 2009.

[5] R. D. Gomes et al.. "Estimação de qualidade de enlace e alocação dinâmica de canais em redes de sensores sem fio industriais." 2017.

[6] R. Delgado Gomes, M. Oliveira Adissi, A. Cavalcante Lima-Filho, M. A Spohn and F. Antônio Belo. "On the Impact of Local Processing for Motor Monitoring Systems in Industrial Environments Using Wireless Sensor Networks". International Journal of Distributed Sensor Networks, vol. 9, no. 7, pp. 471917, 2013.

[7] R. D. Gomes, M. S. Alencar, I. E. Fonseca and A. C. Lima Filho "Desafios de redes de sensores sem fio industriais". Revista de Tecnologia da Informação e Comunicação, vol. 4, no. 1, pp. 16-27, 2014.

[8] R. D. Gomes, M. A. Spohn, A. C. Lima, E. G. dos Anjos and F. A. Belo. "Correlation between spectral occupancy and packet error rate in ieee 802.15. 4-based industrial wireless sensor networks". IEEE Latin America Transactions, vol. 10, no. 1, pp. 1312-1318, 2012.

[9] A. Goldsmith. Wireless communications. Cambridge university press, 2005.

[10] T. S. Rappaport. Comunicações Sem Fio. Pearson Education, 2009. Segunda Edição.

[11] "IEEE Standard for Low-Rate Wireless Networks". IEEE Std 802.15.4-2015 (Revision of IEEE Std 802.15.4-2011), pp. 1- $\overline{709 \text {, April }}$ 2016.

[12] P. Tuset-Peiró, R. D. Gomes, P. Thubert, E. Cuerva, E. Egusquiza and X. Vilajosana. "A Dataset to Evaluate IEEE 802.15.4g SUN for Dependable Low-Power Wireless Communications in Industrial Scenarios". Data, vol. 5, no. 3, pp. 64, Jul 2020.

[13] D. Medeiros and D. Gomes, Ruan. "Repositório GitHub". https://github.com/DiegomMedeiros/IEEE802154g-adaptivemodulation, 2021 